\title{
Bladder cancer: do we need contrast injection for MRI assessment of muscle invasion? A prospective multi-reader VI-RADS approach
}

\author{
Andrea Delli Pizzi ${ }^{1,2}$ (D) $\cdot$ Domenico Mastrodicasa $^{3} \cdot$ Michele Marchioni $^{4,5} \cdot$ Giulia Primiceri $^{4} \cdot$ Francesca Di Fabio $^{1}$. \\ Roberta Cianci ${ }^{1}$ - Barbara Seccia ${ }^{1}$ - Barbara Sessa ${ }^{1}$ • Erica Mincuzzi ${ }^{1}$ - Martina Romanelli ${ }^{1}$ - Pietro Castellan ${ }^{4}$. \\ Roberto Castellucci $^{4}$ - Antonella Colasante ${ }^{6}$ - Luigi Schips $^{4}$ - Raffaella Basilico ${ }^{1}$ - Massimo Caulo ${ }^{1,2}$
}

Received: 7 August 2020 / Revised: 12 October 2020 / Accepted: 4 November 2020 / Published online: 19 November 2020

(C) The Author(s) 2020

\begin{abstract}
Objectives (1) To investigate whether a contrast-free biparametric MRI (bp-MRI) including T2-weighted images (T2W) and diffusion-weighted images (DWI) can be considered an accurate alternative to the standard multiparametric MRI (mp-MRI), consisting of T2, DWI, and dynamic contrast-enhanced (DCE) imaging for the muscle-invasiveness assessment of bladder cancer (BC), and (2) to evaluate how the diagnostic performance of differently experienced readers is affected according to the type of MRI protocol.

Methods Thirty-eight patients who underwent a clinically indicated bladder mp-MRI on a 3-T scanner were prospectively enrolled. Trans-urethral resection of bladder was the gold standard. Two sets of images, set 1 (bp-MRI) and set 2 (mp-MRI), were independently reviewed by four readers. Descriptive statistics, including sensitivity and specificity, were calculated for each reader. Receiver operating characteristic (ROC) analysis was performed, and the areas under the curve (AUCs) were calculated for the bp-MRI and the standard mp-MRI. Pairwise comparison of the ROC curves was performed.

Results The AUCs for bp- and mp-MRI were respectively 0.91-0.92 (reader 1), 0.90 (reader 2), 0.95-0.90 (reader 3), and 0.900.87 (reader 4). Sensitivity was $100 \%$ for both protocols and specificity ranged between 79.31 and $89.66 \%$ and between 79.31 and $83.33 \%$ for bp-MRI and mp-MRI, respectively. No significant differences were shown between the two MRI protocols $(p>0.05)$. No significant differences were shown accordingly to the reader's experience $(p>0.05)$.

Conclusions A bp-MRI protocol consisting of T2W and DWI has comparable diagnostic accuracy to the standard mp-MRI protocol for the detection of muscle-invasive bladder cancer. The experience of the reader does not significantly affect the diagnostic performance using VI-RADS.

Key Points

- The contrast-free MRI protocol shows a comparable accuracy to the standard multiparametric MRI protocol in the bladder cancer muscle-invasiveness assessment.

- VI-RADS classification helps non-expert radiologists to assess the muscle-invasiveness of bladder cancer.

- DCE should be carefully interpreted by less experienced readers due to inflammatory changes representing a potential pitfall.
\end{abstract}

Keywords Bladder cancer $\cdot$ Magnetic resonance imaging $\cdot$ Diffusion magnetic resonance imaging $\cdot$ Contrast media

Andrea Delli Pizzi

andreadellipizzi@gmail.com

1 Department of Neuroscience, Imaging and Clinical Sciences, "G. d'Annunzio" University, Via Luigi Polacchi 11, 66100 Chieti, Italy

2 ITAB - Institute of Advanced Biomedical Technologies, "G. d'Annunzio" University, Chieti, Italy
3 Department of Radiology, Stanford University School of Medicine, Stanford, CA, USA

4 Department of Medical, Oral and Biotechnological Sciences, Urology Unit, SS Annunziata Hospital, G. d'Annunzio University of Chieti, Chieti, Italy

5 Laboratory of Biostatistics, Department of Medical, Oral and Biotechnological Sciences, Chieti, Italy

6 Surgical Pathology Unit, SS Annunziata Hospital, Chieti, Italy 


$\begin{array}{ll}\text { Abbreviations } \\ \text { ADC } & \text { Apparent diffusion coefficient } \\ \text { BC } & \text { Bladder cancer } \\ \text { bp-MRI } & \text { Biparametric magnetic resonance imaging } \\ \text { DCE } & \text { Dynamic contrast-enhanced } \\ \text { DWI } & \text { Diffusion-weighed imaging } \\ \text { MIBC } & \text { Muscle-invasive bladder cancer } \\ \text { mp-MRI } & \text { Multiparametric magnetic resonance imaging } \\ \text { NMIBC } & \text { Non-muscle-invasive bladder cancer } \\ \text { T2W } & \text { T2W-weighted images } \\ \text { TURB } & \text { Trans-urethral resection of bladder } \\ \text { VI-RADS } & \text { Vesical Imaging-Reporting and Data System }\end{array}$

\section{Introduction}

Bladder cancer (BC) is a leading cause of cancer-related death in men and accounts for approximately 550,000 new cases per year worldwide [1]. About a quarter of BCs are muscleinvasive (MIBC). MIBCs are $>\mathrm{T} 1$ tumors requiring radical cystectomy, with or without neo- or adjuvant chemotherapy, and have significant impact on the patient survival [2]. On the other hand, non-muscle-invasive bladder cancers (NMIBCs), representing Ta-T1 tumors, are frequently treated locally and are characterized by a better prognosis [3]. Although transurethral resection of bladder (TURB) represents the gold standard for BC local staging, it has some limitations. In fact, it is burdened with a high risk of under-staging in the presence of multiple or large lesions and in T1 tumors potentially requiring a second TURB, causing a delay in further treatments and overall increased healthcare costs [4-6].

Multiparametric magnetic resonance imaging (mp-MRI) has recently become an emerging method for the $\mathrm{BC}$ local staging and, in particular, for the detection of MIBC [7-9]. In this context, the Vesical Imaging-Reporting and Data System (VI-RADS), introduced in 2018, represents a standardized reporting criterion for bladder MRI aiming to improve the communication among doctors, thus potentially improving patient management [8]. In fact, recent studies demonstrated high accuracy of VI-RADS for discriminating MIBC and NMIBC with a good inter-reader agreement [10-14]. More in detail, the recommended image protocol includes conventional T2W-weighted images (T2W), diffusion-weighed imaging (DWI), and dynamic contrastenhanced (DCE) imaging. Among these sequences, DWI was considered the dominant sequence to estimate the muscle invasion [8]. Recent studies demonstrated the feasibility of contrast-free MR protocols for the local staging of several tumors, including rectal cancer and prostate cancer, with promising results. However, to the best of our knowledge, no studies compared the standard $\mathrm{mp}$ - and a contrast-free MRI protocol (for the VI-RADS assessment) $[15,16]$. In this setting, scan time and costs would be reduced. Moreover, patient safety and comfort would be improved, avoiding the risks related to the intravenous administration of gadoliniumbased contrast agents, such as nephrogenic systemic fibrosis, renal failure, and tissue accumulation of gadolinium.

We hypothesized that a non-contrast, biparametric magnetic resonance imaging (bp-MRI) might be useful to assess BC local staging. The purpose of this prospective study was to investigate whether a contrast-free bp-MRI, including T2W and DWI, can be considered an accurate alternative to the standard mp-MRI for the muscle-invasiveness assessment of $\mathrm{BC}$, using a 3-T MR scanner. Moreover, we explored how these two different protocols affect the diagnostic performance when they are assigned to four readers with different expertise in abdominal imaging.

\section{Materials and methods}

\section{Patient population and study design}

This prospective study received formal Institutional Review Board and Ethical Committee approval. The study was conducted in line with the European Urology and Good Clinical Practice guidelines according to ethical principles laid down by the latest version of the Declaration of Helsinki. Written informed consent was obtained from all patients enrolled in the study [4]. A total of 41 patients who underwent clinically indicated multiparametric MRI (mp-MRI) between August 2019 and December 2019 were prospectively included (Fig. 1). Inclusion criteria were (1) biopsy-proved urothelial BC after cystoscopy, (2) mp-MRI of bladder performed using a 3-T scanner, and (3) TURB with histological evaluation. Finally, 3 patients were excluded: one patient for severe susceptibility artifacts in the pelvis (hip replacement), one because the MR exam was performed on a 1.5-T scanner, and another patient had contraindications to intravenous contrast administration. The final study population consists of 38 patients.

\section{MRI protocol}

A state-of-the-art 3-T MR scanner (dStream, Philips Medical System) equipped with a phased array surface coil was used in this study. The MR protocol included the following sequences: T2W turbo spin-echo images, DWI, and DCE T1weighted 3D spoiled gradient-echo images (DCE). A more detailed description of the MR parameters is summarized in Table 1. Concerning DWI, four $b$-values $(b=600, b=1000, b$ $=1500$, and $b=2000$ ) were acquired and apparent diffusion coefficient maps (ADC maps) were calculated for each patient. Gadoteridol (Prohance; Bracco) was used in a dose of $0.2 \mathrm{mmol} / \mathrm{kg}$ (flow rate of $2 \mathrm{~mL} / \mathrm{s}$ ). Patients without contraindications received $20 \mathrm{mg}$ of scopolamine butylbromide 
Fig. 1 Study flowchart

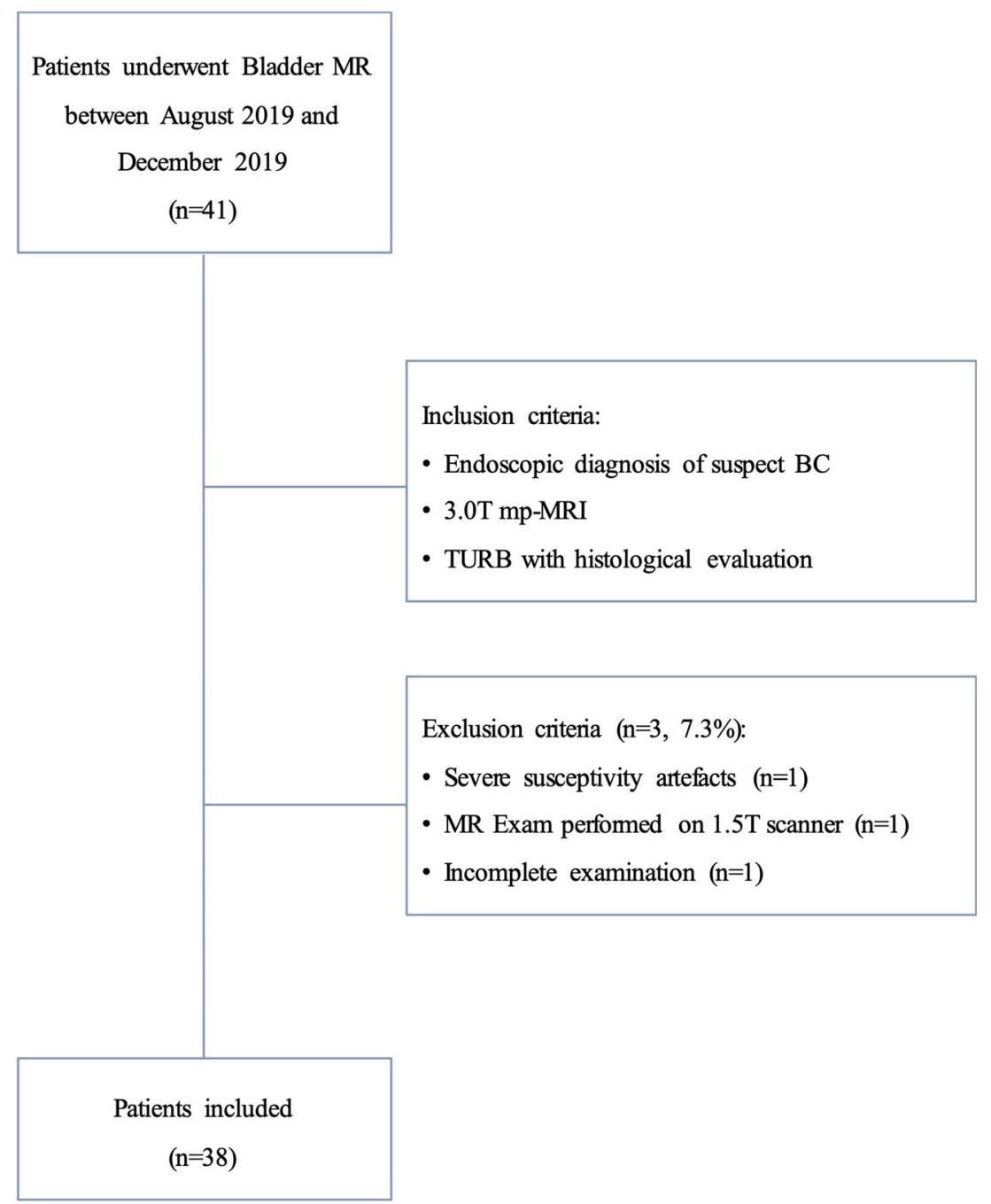

(Buscopan, Boehringer Ingelheim) intravenously to reduce the incidence of motion artifacts due to bowel motility. Patients were instructed to void $1-2 \mathrm{~h}$ before imaging or to start drinking $500-1000 \mathrm{~mL}$ of water in the $30 \mathrm{~min}$ before the examination, depending on the patient's tolerance level. The degree of bladder filling was evaluated using ultrasound

Table 1 Parameters of the T2weighted, DWI, and DCE sequences included in the study protocol

\begin{tabular}{llll}
\hline & T2-weighted fast spin-echo & DWI* & DCE \\
\hline Image set 1 & Yes & Yes & No \\
Image set 2 & Yes & Yes & Yes \\
Repetition time (msec) & $3000-5000$ & 3000 & 3.1 \\
Echo time (msec) & 80 & 97 & 1.42 \\
Matrix & $200 \times 179$ & $68 \times 54$ & $100 \times 139$ \\
Flip angle & 90 & 90 & 10 \\
Number of excitations & 2 & $3-12$ & 2 \\
Section thickness (mm) & 4 & 4 & 4 \\
Imaging planes & Transverse ${ }^{\dagger}$, coronal, sagittal & Transverse $\dagger$, sagittal & Transverse \\
Acquisition time (min) & 2.26 & 4.19 & 3.26 \\
\hline
\end{tabular}

DWI sequences included ADC map calculation

*DWI performed with $b$-values of 0, 600, 1000, 1500, and $2000 \mathrm{~s} / \mathrm{mm}$

${ }^{\dagger}$ Transverse plane angulated perpendicularly to long axis of bladder 
before the patient enters the MR room. MR was performed with patient in supine position [8].

\section{Image analysis}

Two sets of images, namely set 1 and set 2 , were evaluated on a dedicated post-processing workstation (Vue Picture Archiving and Communication System, version 12.2, Carestream Health). Set 1 (non-contrast protocol) was presented in the first reading session and included the biparametric MRI (bp-MRI) protocol consisting of

a) Axial, sagittal, and coronal $\mathrm{T} 2 \mathrm{~W}$ images;

b) Axial and sagittal DWI images (including the corresponding ADC maps).

Set 2 (full protocol) was assigned to the second reading session and included set 1 images in addition to DCE images. Both sets of images were independently reviewed by four readers with different degrees of experience in abdominal radiology ( 2 expert radiologists with 10 and 15 years respectively in abdominal radiology, 1 senior resident, and 1 secondyear resident), in two reading sessions separated by at least 4 weeks, to avoid recall bias. MRI criteria for muscleinvasiveness were assessed according to VI-RADS and bladder subdivisions in sectors $[8,14]$. In this way, twelve sectors were considered. According to VI-RADS, all detected lesions were scored on a 5-point scale based on the likelihood of muscle invasion: 1 , highly unlikely; 2 , unlikely; 3 , equivocal; 4 , likely; 5, very likely [8]. More in detail, the presence of muscle invasion was suspected on the basis of the following criteria:

- T2W: interruption of low signal intensity line of the muscular layer (likely) or extension of the intermediate signal intensity tumor to the extra-vesical fat (very likely);

- DWI: focal extension of high signal intensity tumor on DWI and low signal intensity on ADC to the muscular layer (likely) or to the entire bladder wall and extravesical fat (very likely);

- DCE: focal extension of tumor early enhancement to the muscular layer (likely) or to the entire bladder wall and extra-vesical fat (very likely) [8].

\section{Reference standard}

The urologic evaluation included the description of lesion number, size, morphology, and location. A standardized form with a descriptive schematic map was used for cystoscopy, MRI, and TURB, to record all the information on each lesion. More in detail, according to Marchioni et al, bladder was divided in ten regions: trigone, right ureteral orifice, left ureteral orifice, right wall, left wall, anterior wall, posterior wall, dome, neck, and posterior urethra [14]. All patients underwent a standard TURB and a piecemeal resection in fraction $[4,10,11,17-20]$. The base of each lesion was sent to histological evaluation separately with a numerical code to compare histological results with those of cystoscopy and mpMRI. Once the muscle-invasiveness was histologically proved, patients underwent to radical cystectomy. Since they were eligible, they also received a platinum-based neoadjuvant chemotherapy [21]. When indicated, a second TURB was performed according to the EAU guidelines [4]. Specimens were examined to assess the type, grade, and stage of the tumor. The World Health Organization classification was used to classify and grade malignant tumors, while the American Joint Committee on Cancer/Union for International Cancer Control TNM system was used to stage each lesion [4].

\section{Statistical analysis}

The Shapiro-Wilk test was used to test the distribution of quantitative variables showing a non-normal distribution. Sample size estimation was performed considering the association between VI-RADS score and MIBC status. The reference was the proportion of NMIBC and MIBC stratified according to VI-RADS score in previous studies $[14,19]$. A power of $90 \%$ and a value of $5 \%$ were considered for the $\chi^{2}$ test. Sample size was estimated by using the R package "pwr" (version 1.2.2; function: pwr.chisq.test). The estimated effect size was 0.7 , and the number of patients needed to obtain the desired power was 31 subjects. A dichotomization of VIRADS scores was performed. In detail, concerning the diagnostic accuracy for the detection of MIBC, VI-RADS scores of 1-3 were considered negative for muscle-invasiveness, while VI-RADS scores of 4 and 5 were considered positive.

Descriptive statistics, including sensitivity and specificity, were calculated for each reader. Receiver operating characteristic (ROC) analysis was performed, and areas under the curve (AUCs) were calculated for each reader and image set. A comparison of ROC curves was performed to test the difference between the areas under the ROC curves among the four readers. ROC curve comparison was performed with MedCalc software, version 16.8.4 (MedCalc Software). All other statistical tests were performed by using IBM SPSS Statistics software, version 20 (IBM). A $p$ value $\leq 0.05$ was considered statistically significant.

\section{Results}

Most of the 38 patients included in the study were male (27, $71.1 \%$ ), and the median age was 72.5 (IQR: 66.5-81.0) years (Table 2). Out of the 38 patients, 7 (18\%) had a MIBC (T2- 
Table 2 Descriptive baseline characteristics of included patients $(n=$ 38). Continuous variables are summarized as median and interquartile ranges (IQR). Categorical variables are summarized as frequencies and percentages $(\%)$

\begin{tabular}{ll}
\hline Features & Value \\
\hline Age & $72.5(66.5,81.0)$ \\
Gender, male & $27(71.4 \%)$ \\
Body mass index, $\mathrm{kg} / \mathrm{m}^{2}$ & $26.6(24.0,29.1)$ \\
Smoking history & $14(36.8 \%)$ \\
Previous bladder cancer & $19(50.0 \%)$ \\
Charlson Comorbidity Index & \\
0 & $21(55.3 \%)$ \\
1 & $13(34.2 \%)$ \\
2 & $3(7.9 \%)$ \\
3 & $1(2.6 \%)$ \\
Urine cytology & \\
Non-diagnostic & $13(34.2 \%)$ \\
Negative & $16(42.1 \%)$ \\
Positive & $9(23.7 \%)$ \\
Previous endovesical treatment & \\
Bacillus Calmette-Guerin & $4(10.5 \%)$ \\
Epirubicin & $1(2.6 \%)$ \\
Mitomycin C & $3(7.9 \%)$ \\
None & $30(78.9 \%)$ \\
\hline
\end{tabular}

T3), and $31(82 \%)$ had a NMIBC (Ta-T1). A total of $68 \mathrm{BCs}$ were found. More in detail, 33 (48.5\%) were Ta, 28 (41.2\%) were T1, $6(8.8 \%)$ were $\mathrm{T} 2 \mathrm{~W}$, and $1(1.5 \%)$ was T3. All readers correctly classified the 7 MIBC patients with both MRI protocols.

The diagnostic performance of the readers referring to descriptive statistics and ROC analysis is shown in Table 3. More in detail, no significant differences were shown in diagnostic performance for all readers between the two protocols $(p>0.05)$. In fact, the AUC for the bp- and mp-MRI protocol was respectively $0.91-0.92$ (reader 1 ), 0.90 (reader 2), $0.95-$ 0.90 (reader 3), and $0.90-0.87$ (reader 4). No significant differences in diagnostic performance were found among the four readers in the pairwise comparisons $(p>0.05)$.

Table 4 shows a per-lesion diagnostic performance analysis for each reader to classify MIBC according to the TNM stage classification correctly. Comparing the false positive cases between bp-MRI and mp-MRI, they reduced for reader 1 (from 7 to 6), were stable for reader 2 (7), and increased for the two less experienced readers when using the mp-MRI (from 4 to 7 for reader 3 , from 8 to 10 for reader 4 ). The number of false negative cases remained stable for readers 2 and 3 ( 6 cases for both readers) and reduced when using the mp-MRI for readers 1 and 4 (from 7 to 6 and from 2 to 1 , respectively). Case examples of correctly and incorrectly classified MIBC are shown in Figs. 2 and 3, respectively.

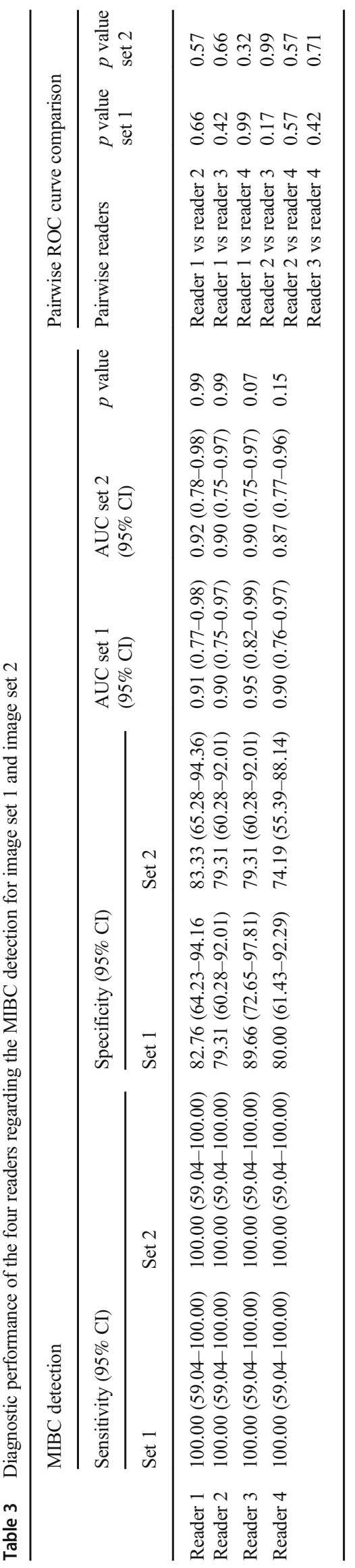


Table 4 Per-lesion diagnostic performance of the four readers to correctly classify MIBC according to TNM stage classification

\begin{tabular}{|c|c|c|c|c|c|c|c|}
\hline & & \multicolumn{3}{|c|}{ Biparametric bladder MR } & \multicolumn{3}{|c|}{ Triparametric bladder MR } \\
\hline & & \multirow[t]{2}{*}{ Correctly classified } & \multicolumn{2}{|c|}{ Incorrectly classified } & \multirow[t]{2}{*}{ Correctly classified } & \multicolumn{2}{|c|}{ Incorrectly classified } \\
\hline & & & False negatives & False positives & & False negatives & False positives \\
\hline \multirow[t]{4}{*}{$\mathrm{Ta}(n=33)$} & Reader 1 & 25 & 5 & 3 & 26 & 4 & 3 \\
\hline & Reader 2 & 26 & 3 & 4 & 26 & 3 & 4 \\
\hline & Reader 3 & 26 & 3 & 4 & 25 & 3 & 5 \\
\hline & Reader 4 & 27 & 2 & 4 & 26 & 1 & 6 \\
\hline \multirow[t]{4}{*}{$\mathrm{T} 1(n=28)$} & Reader 1 & 24 & 0 & 4 & 25 & 0 & 3 \\
\hline & Reader 2 & 22 & 3 & 3 & 22 & 3 & 3 \\
\hline & Reader 3 & 25 & 3 & 0 & 23 & 3 & 2 \\
\hline & Reader 4 & 24 & 0 & 4 & 24 & 0 & 4 \\
\hline \multirow[t]{4}{*}{$\mathrm{T} 2 \mathrm{~W}(n=6)$} & Reader 1 & 6 & 0 & 0 & 6 & 0 & 0 \\
\hline & Reader 2 & 6 & 0 & 0 & 6 & 0 & 0 \\
\hline & Reader 3 & 6 & 0 & 0 & 6 & 0 & 0 \\
\hline & Reader 4 & 6 & 0 & 0 & 6 & 0 & 0 \\
\hline \multirow[t]{4}{*}{$\mathrm{T} 3(n=1)$} & Reader 1 & 1 & 0 & 0 & 1 & 0 & 0 \\
\hline & Reader 2 & 1 & 0 & 0 & 1 & 0 & 0 \\
\hline & Reader 3 & 1 & 0 & 0 & 1 & 0 & 0 \\
\hline & Reader 4 & 1 & 0 & 0 & 1 & 0 & 0 \\
\hline
\end{tabular}

\section{Discussion}

Our results demonstrate that a contrast-free MR protocol of the bladder has the same diagnostic accuracy of the standard mp-MRI for assessing BC's muscle-invasiveness, regardless of the reader's experience.

These results firstly confirm the relevance of DWI in the risk evaluation of muscle-invasiveness in bladder cancer [8]. In detail, the diagnostic accuracy of the bp-MRI protocol is in line with what is reported by several other studies [22-26]. Secondly, our results support the promising results of Barchetti et al, on the VI-RADS repeatability [11]. In fact, in our study, the diagnostic performance of all readers was substantially comparable, demonstrating that VI-RADS is a useful tool even in less experienced readers.

An interesting finding was the worse diagnostic performance of the two less experienced readers (readers 3 and 4) when including DCE imaging. On the other hand, the diagnostic performance of the two most experienced readers was not significantly affected between the two protocols. More in detail, focusing on the per-lesion muscle-invasiveness assessment (Table 4), we observed that, while the overall number of false negative cases remained stable among the four readers, the overall number of false positive cases increased in most readers when adding DCE imaging. The overall increase was $15.4 \%$ (from 26 to 30 ) in line with what is reported in other studies $[22,27,28]$. However, considering only readers 3 and 4 , it reached $41.7 \%$ (from 12 to 17 ), implying that DCE imaging should be interpreted carefully by not-expert readers. We believe there are two possible reasons. First, we postulated that DWI imaging could require a relatively more straightforward learning curve compared to DCE imaging. For instance, the general principle of DWI is relatively straightforward: the tumor appears as a hyperintense signal interrupting or not the submucosal muscle, which has a typical low-intermediate signal intensity. Moreover, as demonstrated in pelvic tumors, our DWI protocol includes high $b$-values, which could have improved the diagnostic confidence of the less experienced readers by providing a better background suppression [29, 30]. Secondly, it is well known that, in approximately $60 \%$ of DCE studies, the submucosa and the tumor show similar signal intensity, thus making more challenging the detection of the submucosal linear enhancement [31]. Moreover, perivesical enhancement due to reactive or acute inflammatory changes, for instance, in case of concomitant cystitis, may be a confounding factor potentially leading to an overestimation of the tumor stage [28, 32].

Despite these results, the mp-MRI protocol reduced the overall number of false negative cases among the four readers (from 21 to 19). In detail, only adding DCE, two readers recognized a missed 3-mm Ta BC. In this regard, further studies are necessary to investigate the added value of DCE in $<5$ mm lesions.

A potential downside of bp-MRI of the bladder is that DWI has few potential pitfalls that may affect the diagnostic accuracy [33]. For example, the presence of edema can be 


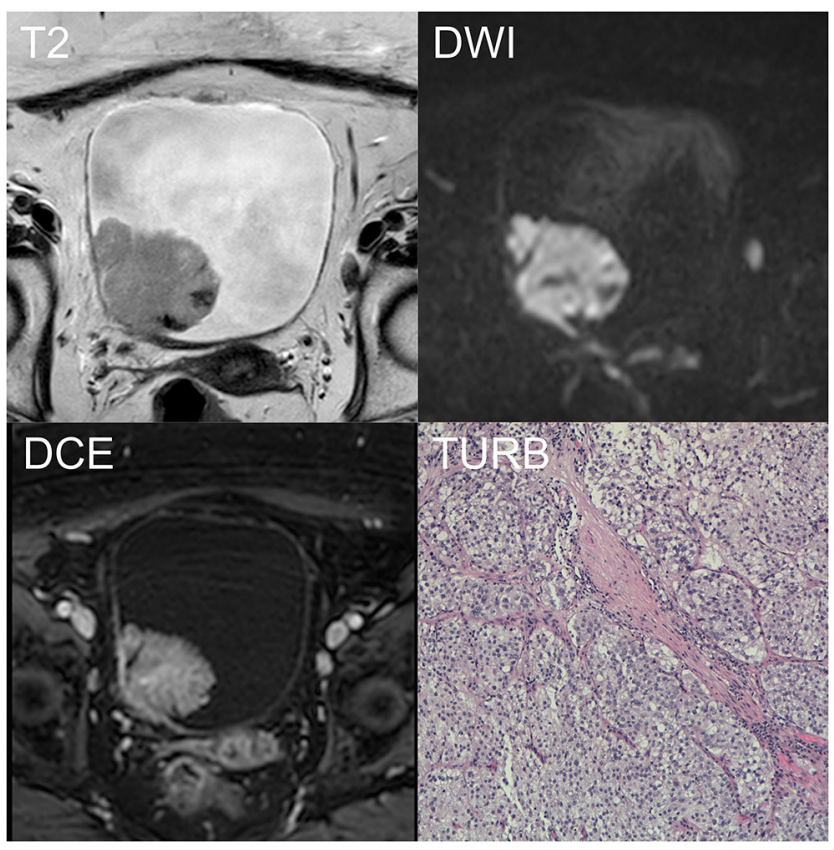

Fig. 2 Example of correctly classified muscle-invasive BC. A 90-yearold woman with hematuria and a bladder mass reported after flexible cystoscopy underwent mp-MRI before primary TURB. T2W imaging (T2) showing an exophytic lesion on the right lateral wall, $>1 \mathrm{~cm}$ in the major axis with focal interruption of the SI of the muscularis propria. The b1000-DWI (DWI) confirming the high signal intensity of the tumor extending to the muscular layer. Based on bp-MRI, all readers assigned a VI-RADS category of 4. DCE imaging (DCE) showing early enhancement of the lesion and inner layer with an early enhancement of the muscularis propria, indicating tumor infiltration. DCE did not affect the VI-RADS category. T stage after TURB was HG-T2 (TURB). DCE, dynamic contrast-enhanced; DWI, diffusion-weighted imaging; HG, high grade; mp-MRI, multiparametric magnetic resonance imaging; bp-MRI, biparametric magnetic resonance imaging; SI, signal intensity; T2W, T2weighted; TURB, trans-urethral resection of the bladder; VI-RADS, Vesical Imaging-Reporting and Data System

responsible for high signal intensity on DWI due to the intrinsic long T2W decay time of water (also known as "shinethrough" effect) [34]. To overcome this problem, we recommend using multiple $b$-values including middle high $b$-values (b800-b1000) and very high $b$-values (b1500-b2000), where the shine-through effect is more likely to be absent, and to include ADC map on visual analysis.

Although it is a relatively novel topic in bladder cancer, the value of a contrast-free protocol was already investigated in the pelvis. For example, Di Campli et al reported no significant differences between the standard multiparametric and biparametric (T2W and DWI) MRI for clinically significant prostate cancer detection [16]. Concerning rectal cancer, the 2016 consensus meeting of the European Society of Gastrointestinal and Abdominal Imaging recommended the crucial role of DWI in the staging and the re-staging after chemoradiotherapy and before surgery $[15,35]$.

To the best of our knowledge, this is the first study comparing the diagnostic accuracy of bladder bp-MRI and mp-

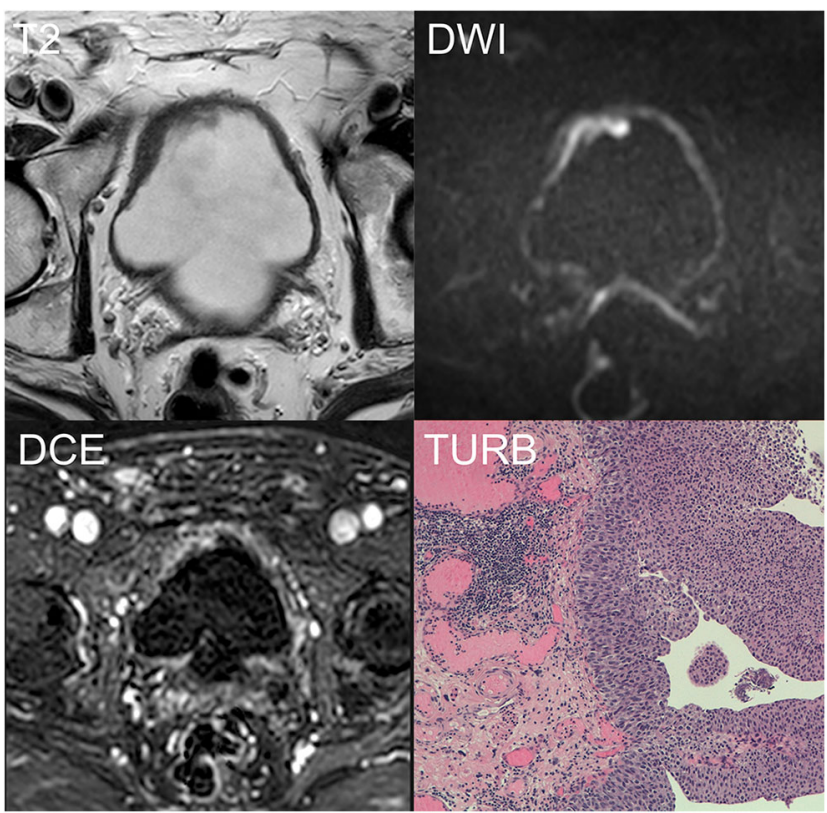

Fig. 3 Example of incorrectly classified not muscle-invasive BC. An 89year-old man with hematuria and bladder mass reported after flexible cystoscopy underwent mp-MRI before primary TURB. T2W imaging (T2) shows a slightly exophytic lesion on the right antero-lateral wall, which is $1 \mathrm{~cm}$ thick with equivocal interruption of the SI of the muscularis propria. The $b 1000$-DWI (DWI) clearly demonstrated the continuous intermediate signal intensity of the muscular layer. Based on bp-MRI, three of the four readers assigned a VI-RADS category of 3. DCE imaging (DCE) showing early enhancement of the lesion with an inhomogeneous enhancement of the underlying inner layer due to inflammatory changes. This finding was misinterpreted by two readers as the muscularis propria infiltration, thus modifying the VI-RADS category from 3 to 4 . T stage after TURB was LG-T1 (TURB). After 4 weeks, re-TURB was performed confirming the absence of residual tumor. DCE, dynamic contrast-enhanced; DWI, diffusion-weighted imaging; LG, low grade; mp-MRI, multiparametric magnetic resonance imaging; bp-MRI, biparametric magnetic resonance imaging; SI, signal intensity; T2W, T2weighted; TURB, trans-urethral resection of the bladder; re-TURB, repeated trans-urethral resection of the bladder; VI-RADS, Vesical Imaging-Reporting and Data System

MRI for the muscle-invasiveness assessment using VIRADS in a clinical setting. Most of the recent studies focused on standard mp-MRI and the feasibility of VI-RADS [11-13]. In addition, our multi-reader approach may expand the applicability of our results to clinical settings with less experienced readers. In light of the emerging role of VI-RADS and its potential future extension to the surveillance and treatment response assessment, the results of our study may have a beneficial impact on costs, scan time, and patient safety [36, 37].

Overall, from a clinical point of view, it should be pointed out that currently to think to a patient management relying only on MRI findings is not realistic. In fact, although we had no "false negative" muscle-invasive bladder cancers in our study, this event may produce a rapid progression and spread of the tumor with devastating consequences for the patient. Similarly, to perform a radical cystectomy in a "false positive" muscle-invasive bladder cancer would be a failure as 
well, especially considering the high comorbidity rate of this surgical treatment. For these reasons, as recently discussed by Marchioni et al, a probable scenario would be to consider MRI in a more complex and articulate approach to the patient, in order to reduce useless cystoscopy or re-TURB [14].

Our study has some limitations. First of all, the type of VIRADS scores dichotomization could have represented a bias. In fact, the choice of the appropriate VI-RADS category to define whether the tumor is muscle-invasive is controversial $[14,38]$. Marchioni et al recently demonstrated that a threshold of 4 significantly improved MIBC detection, reaching an accuracy of $90 \%$. Moreover, the type of dichotomization and the relatively small study cohort could have affected the sensitivity, which is higher than other studies [14, 38, 39]. Secondly, patient selection in the study was widely influenced by physician experience and patient characteristics. In this way, patients with worrisome tumor features (i.e., large and solid tumors) may have not been included to avoid delaying cystectomy. Third, ours is a single-center study. However, all the studies recently published involved a single center. Therefore, further studies, possibly including multiple institutions, shall represent the next step for future standardization $[11,14,19,38,39]$. Nonetheless, we used a state-of-the-art 3T MR scanner for all patients, thus setting up a good standard for future investigation. In fact, 3-T MRI has been proven to provide better specificity and sensitivity for the bladder cancer $\mathrm{T}$ staging [40]. Fourth, we know that a certain degree of unbalanced data (patients and lesions, MIBC vs. NMIBC) could have affected our results. However, considering the purpose of this study, our primary objective was to test if any difference in MIBC detection at biparametric MRI vs. multiparametric MRI exists. In this way, all lesions were examined with both protocols by different readers. Moreover, both a per-patient and a per-lesion analyses were performed for each reader.

\section{Conclusion}

A contrast-free MR imaging protocol consisting of T2W and DWI has comparable diagnostic accuracy to that of a standard mp-MR imaging protocol for the detection of MIBC. Moreover, considering both MR protocols, the experience of the reader does not significantly affect the diagnostic performance when using VI-RADS classification. Further validation studies in a larger population are warranted to define better the clinical role of bp-MRI, and whether it could be considered an accurate alternative to the standard protocol definitively.

Acknowledgments The authors thank Darien Calvo Garcia for his insightful contribution on the MR protocol setting and the acquisition of data.
Funding Open access funding provided by Università degli Studi G. D'Annunzio Chieti Pescara within the CRUI-CARE Agreement.

\section{Compliance with ethical standards}

Guarantor The scientific guarantor of this publication is Andrea Delli Pizzi.

Conflict of interest The authors of this manuscript declare no relationships with any companies whose products or services may be related to the subject matter of the article.

Statistics and biometry No complex statistical methods were necessary for this paper.

Informed consent Written informed consent was obtained from all subjects (patients) in this study.

Ethical approval All procedures were performed in accordance with the ethical standards of the institutional research committee (Comitato di Etica per la Ricerca Biomedica delle province di Chieti e Pescara e dell'Università degli Studi "G.d'Annunzio" di Chieti e Pescara, no. 1767) and with the 1964 Declaration of Helsinki and its later amendments or comparable ethical standards. Informed consent was obtained from each participant.

Study subjects or cohorts overlap The study subjects have been previously reported in the following study:

Marchioni, M., et al, Could bladder multiparametric MRI be introduced in routine clinical practice? Role of the new VI-RADS score: results from a prospective study. Clin Genitourin Cancer, 2020.

\section{Methodology \\ - prospective \\ - diagnostic study, observational \\ - performed at one institution}

Open Access This article is licensed under a Creative Commons Attribution 4.0 International License, which permits use, sharing, adaptation, distribution and reproduction in any medium or format, as long as you give appropriate credit to the original author(s) and the source, provide a link to the Creative Commons licence, and indicate if changes were made. The images or other third party material in this article are included in the article's Creative Commons licence, unless indicated otherwise in a credit line to the material. If material is not included in the article's Creative Commons licence and your intended use is not permitted by statutory regulation or exceeds the permitted use, you will need to obtain permission directly from the copyright holder. To view a copy of this licence, visit http://creativecommons.org/licenses/by/4.0/.

\section{References}

1. Bray F, Ferlay J, Soerjomataram I, Siegel RL, Torre LA, Jemal A (2018) Global cancer statistics 2018: GLOBOCAN estimates of incidence and mortality worldwide for 36 cancers in 185 countries. CA Cancer J Clin 68:394-424

2. Richters A, Aben KKH, Kiemeney L (2019) The global burden of urinary bladder cancer: an update. World J Urol. https://doi.org/10. 1007/s00345-019-02984-4

3. Palumbo C, Mistretta FA, Knipper S et al (2019) How cancerspecific mortality changes over time after radical cystectomy: 
conditional survival of patients with nonmetastatic urothelial carcinoma of the urinary bladder. Urol Oncol 37:893-899

4. Babjuk M, Bohle A, Burger M et al (2017) EAU guidelines on nonmuscle-invasive urothelial carcinoma of the bladder: update 2016. Eur Urol 71:447-461

5. Hurle R, Lazzeri M, Vanni E et al (2018) Active surveillance for low risk nonmuscle invasive bladder cancer: a confirmatory and resource consumption study from the BIAS Project. J Urol 199:401-406

6. Schips L, Augustin H, Zigeuner RE et al (2002) Is repeated transurethral resection justified in patients with newly diagnosed superficial bladder cancer? Urology 59:220-223

7. Zhang N, Wang X, Wang C et al (2019) Diagnostic accuracy of multi-parametric magnetic resonance imaging for tumor staging of bladder cancer: meta-analysis. Front Oncol 9:981

8. Panebianco V, Narumi Y, Altun E et al (2018) Multiparametric magnetic resonance imaging for bladder cancer: development of VI-RADS (Vesical Imaging-Reporting and Data System). Eur Urol 74:294-306

9. Woo S, Panebianco V, Narumi Y et al (2020) Diagnostic performance of Vesical Imaging Reporting and Data System for the prediction of muscle-invasive bladder cancer: a systematic review and meta-analysis. Eur Urol Oncol. https://doi.org/10.1016/j.euo.2020.02.007

10. Del Giudice F, Barchetti G, De Berardinis E et al (2020) Prospective assessment of Vesical Imaging Reporting and Data System (VI-RADS) and its clinical impact on the management of high-risk non-muscle-invasive bladder cancer patients candidate for repeated transurethral resection. Eur Urol 77:101-109

11. Barchetti G, Simone G, Ceravolo I et al (2019) Multiparametric MRI of the bladder: inter-observer agreement and accuracy with the Vesical Imaging-Reporting and Data System (VI-RADS) at a single reference center. Eur Radiol 29:5498-5506

12. Thoeny HC, Bellin MF, Comperat EM, Thalmann GN (2018) Vesical Imaging-Reporting and Data System (VI-RADS): added value for management of bladder cancer patients? Eur Urol 74:307-308

13. Makboul M, Farghaly S, Abdelkawi IF (2019) Multiparametric MRI in differentiation between muscle invasive and non-muscle invasive urinary bladder cancer with Vesical Imaging Reporting and Data System (VI-RADS) application. Br J Radiol 92:20190401

14. Marchioni M, Primiceri G, Delli Pizzi A et al (2020) Could bladder multiparametric MRI be introduced in routine clinical practice? Role of the new VI-RADS score: results from a prospective study. Clin Genitourin Cancer. https://doi.org/10.1016/j.clgc.2020.03.002

15. Delli Pizzi A, Basilico R, Cianci R et al (2018) Rectal cancer MRI: protocols, signs and future perspectives radiologists should consider in everyday clinical practice. Insights Imaging 9:405-412

16. Di Campli E, Delli Pizzi A, Seccia B et al (2018) Diagnostic accuracy of biparametric vs multiparametric MRI in clinically significant prostate cancer: comparison between readers with different experience. Eur J Radiol 101:17-23

17. Witjes JA, Babjuk M, Bellmunt J et al (2020) EAU-ESMO consensus statements on the management of advanced and variant bladder cancer-an international collaborative multistakeholder effort (dagger): under the auspices of the EAU-ESMO Guidelines Committees. Eur Urol 77:223-250

18. Luo C, Huang B, Wu Y, Chen J, Chen L (2020) Use of Vesical Imaging-Reporting and Data System (VI-RADS) for detecting the muscle invasion of bladder cancer: a diagnostic meta-analysis. Eur Radiol 30:4606-4614

19. Del Giudice F, Catto JWF, Panebianco V (2020) Reply to Charalampos Fragkoulis, Georgios Papadopoulos, and Konstantinos Ntoumas's Letter to the Editor re: Francesco Del Giudice, Giovanni Barchetti, Ettore De Berardinis, et al Prospective assessment of Vesical Imaging Reporting and Data System (VI-RADS) and its clinical impact on the management of high-risk non-muscleinvasive bladder cancer patients candidate for repeated transurethral resection. Eur Urol 2020;77:101-9. Eur Urol 77:e94-e95
20. Flaig TW, Spiess PE, Agarwal N et al (2020) Bladder cancer, Version 3.2020, NCCN Clinical Practice Guidelines in Oncology. J Natl Compr Canc Netw 18:329-354

21. Iyer G, Tully CM, Zabor EC et al (2020) Neoadjuvant gemcitabinecisplatin plus radical cystectomy-pelvic lymph node dissection for muscle-invasive bladder cancer: a 12-year experience. Clin Genitourin Cancer. https://doi.org/10.1016/j.clgc.2020.02.014

22. Gupta N, Sureka B, Kumar MM, Malik A, Bhushan TB, Mohanty NK (2015) Comparison of dynamic contrast-enhanced and diffusion weighted magnetic resonance image in staging and grading of carcinoma bladder with histopathological correlation. Urol Ann 7:199-204

23. Takeuchi M, Sasaki S, Ito M et al (2009) Urinary bladder cancer: diffusion-weighted MR imaging-accuracy for diagnosing T stage and estimating histologic grade. Radiology 251:112-121

24. Wu LM, Chen XX, Xu JR et al (2013) Clinical value of T2weighted imaging combined with diffusion-weighted imaging in preoperative $\mathrm{T}$ staging of urinary bladder cancer: a large-scale, multiobserver prospective study on 3.0-T MRI. Acad Radiol 20: 939-946

25. Yamada Y, Kobayashi S, Isoshima S, Arima K, Sakuma H, Sugimura Y (2014) The usefulness of diffusion-weighted magnetic resonance imaging in bladder cancer staging and functional analysis. J Cancer Res Ther 10:878-882

26. Al Johi RS, Seifeldein GS, Moeen AM et al (2018) Diffusion weighted magnetic resonance imaging in bladder cancer, is it time to replace biopsy? Cent European J Urol 71:31-37

27. Kim B, Semelka RC, Ascher SM, Chalpin DB, Carroll PR, Hricak H (1994) Bladder tumor staging: comparison of contrast-enhanced CT, T1- and T2-weighted MR imaging, dynamic gadoliniumenhanced imaging, and late gadolinium-enhanced imaging. Radiology 193:239-245

28. Hassanien OA, Abouelkheir RT, Abou El-Ghar MI, Badawy ME, El Gamal SA, El-Hamid MA (2019) Dynamic contrast-enhanced magnetic resonance imaging as a diagnostic tool in the assessment of tumour angiogenesis in urinary bladder cancer. Can Assoc Radiol J 70:254-263

29. Delli Pizzi A, Caposiena D, Mastrodicasa D et al (2019) Tumor detectability and conspicuity comparison of standard b1000 and ultrahigh b2000 diffusion-weighted imaging in rectal cancer. Abdom Radiol (NY) 44:3595-3605

30. Ueno Y, Kitajima K, Sugimura K et al (2013) Ultra-high b-value diffusion-weighted MRI for the detection of prostate cancer with 3T MRI. J Magn Reson Imaging 38:154-160

31. Tekes A, Kamel I, Imam K et al (2005) Dynamic MRI of bladder cancer: evaluation of staging accuracy. AJR Am J Roentgenol 184: 121-127

32. Rabie E, Faeghi F, Izadpanahi MH, Dayani MA (2016) Role of dynamic contrast-enhanced magnetic resonance imaging in staging of bladder cancer. J Clin Diagn Res 10:TC01-TC05

33. Takeuchi M, Suzuki T, Sasaki S et al (2012) Clinicopathologic significance of high signal intensity on diffusion-weighted MR imaging in the ureter, urethra, prostate and bone of patients with bladder cancer. Acad Radiol 19:827-833

34. Lin WC, Chen JH (2015) Pitfalls and limitations of diffusionweighted magnetic resonance imaging in the diagnosis of urinary bladder cancer. Transl Oncol 8:217-230

35. Beets-Tan RGH, Lambregts DMJ, Maas M et al (2018) Magnetic resonance imaging for clinical management of rectal cancer: updated recommendations from the 2016 European Society of Gastrointestinal and Abdominal Radiology (ESGAR) consensus meeting. Eur Radiol 28:1465-1475

36. Necchi A, Messina A, Briganti A (2018) Re: Valeria Panebianco, Yoshifumi Narumi, Ersan Altun, et al Multiparametric magnetic resonance imaging for bladder cancer: development of VI-RADS (Vesical Imaging-Reporting and Data System). Eur Urol 2018;74: 
294-306: validating VI-RADS criteria within immune-oncology strategies (VI-RADS-IO). Eur Urol 74:e107-e108

37. Necchi A, Bandini M, Calareso G et al (2020) Multiparametric magnetic resonance imaging as a noninvasive assessment of tumor response to neoadjuvant pembrolizumab in muscle-invasive bladder cancer: preliminary findings from the PURE-01 study. Eur Urol 77:636-643

38. Wang H, Luo C, Zhang F et al (2019) Multiparametric MRI for bladder cancer: validation of VI-RADS for the detection of detrusor muscle invasion. Radiology 291:668-674
39. Ueno Y, Takeuchi M, Tamada T et al (2019) Diagnostic accuracy and interobserver agreement for the Vesical Imaging-Reporting and Data System for muscle-invasive bladder cancer: a multireader validation study. Eur Urol 76:54-56

40. van der Pol CB, Chung A, Lim C et al (2018) Update on multiparametric MRI of urinary bladder cancer. J Magn Reson Imaging 48:882-896

Publisher's note Springer Nature remains neutral with regard to jurisdictional claims in published maps and institutional affiliations. 